\title{
Multivariate return periods in hydrology: a critical and practical review focusing on synthetic design hydrograph estimation
}

\author{
B. Gräler ${ }^{1}$, M. J. van den Berg ${ }^{2}$, S. Vandenberghe ${ }^{2}$, A. Petroselli ${ }^{3}$, S. Grimaldi ${ }^{4,5,6}$, B. De Baets ${ }^{7}$, and \\ N. E. C. Verhoest ${ }^{2}$ \\ ${ }^{1}$ Institute for Geoinformatics, University of Münster, Weseler Str. 253, 48151 Münster, Germany \\ ${ }^{2}$ Laboratory of Hydrology and Water Management, Ghent University, Coupure links 653, 9000 Ghent, Belgium \\ ${ }^{3}$ Dipartimento di scienze e tecnologie per l'agricoltura, le foreste, la natura e l'energia (DAFNE Department), \\ University of Tuscia, Via San Camillo De Lellis, 01100 Viterbo, Italy \\ ${ }^{4}$ Dipartimento per la innovazione nei sistemi biologici agroalimentari e forestali (DIBAF Department), University of Tuscia, \\ Via San Camillo De Lellis, 01100 Viterbo, Italy \\ ${ }^{5}$ Honors Center of Italian Universities (H2CU), Sapienza University of Rome, Via Eudossiana 18, 00184 Roma, Italy \\ ${ }^{6}$ Department of Mechanical and Aerospace Engineering, Polytechnic Institute of New York University, \\ Six MetroTech Center Brooklyn, New York, 11201, USA \\ ${ }^{7}$ Department of Mathematical Modelling, Statistics and Bioinformatics, Coupure links 653, 9000 Ghent, Belgium
}

Correspondence to: B. Gräler (ben.graeler@uni-muenster.de)

Received: 10 May 2012 - Published in Hydrol. Earth Syst. Sci. Discuss.: 31 May 2012

Revised: 27 February 2013 - Accepted: 9 March 2013 - Published: 2 April 2013

\begin{abstract}
Most of the hydrological and hydraulic studies refer to the notion of a return period to quantify design variables. When dealing with multiple design variables, the well-known univariate statistical analysis is no longer satisfactory, and several issues challenge the practitioner. How should one incorporate the dependence between variables? How should a multivariate return period be defined and applied in order to yield a proper design event? In this study an overview of the state of the art for estimating multivariate design events is given and the different approaches are compared. The construction of multivariate distribution functions is done through the use of copulas, given their practicality in multivariate frequency analyses and their ability to model numerous types of dependence structures in a flexible way. A synthetic case study is used to generate a large data set of simulated discharges that is used for illustrating the effect of different modelling choices on the design events. Based on different uni- and multivariate approaches, the design hydrograph characteristics of a 3-D phenomenon composed of annual maximum peak discharge, its volume, and duration are derived. These approaches are based on regression analysis, bivariate conditional distributions, bivariate joint distributions and Kendall distribution functions,
\end{abstract}

highlighting theoretical and practical issues of multivariate frequency analysis. Also an ensemble-based approach is presented. For a given design return period, the approach chosen clearly affects the calculated design event, and much attention should be given to the choice of the approach used as this depends on the real-world problem at hand.

\section{Introduction}

A very important objective of hydrological studies is to provide design variables for diverse engineering projects. Recently, there has been an increasing interest in, and need for, simultaneously considering multiple design variables, which are likely to be associated with each other. In hydrology and hydraulics, several applications including sewer systems, dams and flood risk mapping require the selection of storm or hydrograph attributes with a predefined return period.

Standard hydrological design approaches are mostly based on well-established univariate frequency analysis methods. Notwithstanding this, approaches to describe hydrological phenomena involving multiple variables have recently been proposed, aiding the practitioners in estimating multivariate 
return periods. In the literature, as will be described later on, several approaches have evolved over the years. However, it is not clear how these compare to each other and which one is appropriate for a given application.

Recent developments in statistical hydrology have shown the great potential of copulas for the construction of multivariate cumulative distribution functions (CDFs) and for carrying out a multivariate frequency analysis (Favre et al., 2004; Salvadori, 2004; Salvadori and De Michele, 2004, 2007; Salvadori et al., 2007; Genest and Favre, 2007; Salvadori et al., 2011; Vandenberghe et al., 2011). Copulas are functions that combine several univariate marginal cumulative distribution functions into their joint cumulative distribution function. As such, copulas describe the dependence structure between random variables and allow for the calculation of joint probabilities, independently of the marginal behaviour of the involved variables. For more theoretical details, we refer to Sklar (1959) and Nelsen (2006). Several studies have been dedicated to the frequency analysis of multivariate hydrological phenomena such as storms and floods, often within the context of design. However, limited applications have been developed with more than two variables (Vandenberghe et al., 2010; Pinya et al., 2009; Kao and Govindaraju, 2008, 2007; Genest et al., 2007; Serinaldi and Grimaldi, 2007; Zhang and Singh, 2007; Grimaldi and Serinaldi, 2006a,b). For a complete and continuously updated list of papers about copula applications in hydrology see the website of the International Commission on Statistical Hydrology of International Association of Hydrological Sciences ${ }^{1}$.

Multivariate frequency analysis is becoming more and more widespread and several papers provide insight into generalizations of the univariate case and into new definitions of the multivariate return period (see e.g. Salvadori et al., 2011; Salvadori and De Michele, 2004; Shiau, 2003; Yue and Rasmussen, 2002). Since some of the proposed approaches are in contradiction and others are introduced within specific contexts, there exists a need to clarify the definitions provided so far and to highlight their differences. This study is devoted to this issue and compares a set of different approaches on a large simulated data set, allowing illustration of the implications of different modelling choices.

In this paper the construction of multivariate distribution functions based on vine copulas (also referred to as pair-copulas by Aas et al., 2009) is first briefly introduced (Sect. 2.2), followed by an overview of several approaches commonly used to estimate multivariate design events based upon different definitions of joint return periods (JRP) (Sect. 3). Subsequently, a synthetic case study addressing the selection of a design hydrograph is presented, which will serve as a test case for evaluating the different approaches. Section 4 provides all details on the practical context of this case study. Then, in Sect. 5, extreme discharge

\footnotetext{
${ }^{1}$ Available at www.stahy.org.
}

events are selected and their most important variables such as annual maximum peak discharge, its volume, and duration are analysed, as they form the basis of the analysis. Section 6 deals with evaluating the performance and differences between the investigated approaches in quantifying design hydrograph characteristics and highlights important issues for practitioners concerned with multivariate frequency analyses in hydrology. Finally, conclusions are drawn in Sect. 7.

\section{Constructing multivariate copulas}

\subsection{Choice of construction method}

Most of the copula-based research in hydrology addresses the application of 2-D copulas, for which several fitting and evaluation criteria are becoming more and more widespread. In contrast, the use of multidimensional copulas remains a more challenging task. Only a few hydrological studies address this issue and almost always face severe (practical) drawbacks of the available high-dimensional copula families. Most work has been done in the trivariate analysis of rainfall (Zhang and Singh, 2007; Kao and Govindaraju, 2008; Salvadori and De Michele, 2006; Grimaldi and Serinaldi, 2006b), floods (Serinaldi and Grimaldi, 2007; Genest et al., 2007) and droughts (Kao and Govindaraju, 2010; Song and Singh, 2010; Wong et al., 2010).

Recently, a flexible construction method for highdimensional copulas, based on the mixing of (conditional) 2-D copulas, has been introduced and has been shown to have a large potential for hydrological applications. In the literature, this construction is known as the vine copula (or pair-copula) construction (Kurowicka and Cooke, 2007; Aas et al., 2009; Aas and Berg, 2009; Hobæk Haff et al., 2010). The underlying theory for the vine copula construction is described in Bedford and Cooke $(2001,2002)$. This construction method originates from work presented by Joe (1997) on which also the method of conditional mixtures, as applied by De Michele et al. (2007), is based. In this paper the vine copula method will be used to construct the 3-D copula for peak discharge $Q_{\mathrm{p}}$, duration $D$, and volume $V_{\mathrm{p}}$. The construction and fitting is discussed in the next section.

\subsection{Construction of a 3-D vine copula}

In this paper the focus will be on a 3-D vine copula joining the three marginal distributions of three random variables: $X, Y$ and $Z$. In general, the approach can be extended to any number of dimensions, although limitations may be introduced by the computational power and available data. In the following, we assume that the samples of all three variables have each been transformed using the following rank order transformation $S$ in order to obtain the marginal empirical distribution functions:

$S(x):=\frac{\operatorname{rank}(x)}{n+1}$, 
where $n$ denotes the number of observations for the given variable. We denote the transformed variables by $U, V$ and $W$ so that all three variables are now approximately uniformly distributed on $[0,1]$.

The basic idea of vine copulas is to construct highdimensional copulas based on a stagewise mixing of (conditional) bivariate copulas. This corresponds to decomposing the full density function into a product of low-dimensional density functions. At the base of the construction all relevant pairwise dependences are modelled with bivariate copulas. If all mutual dependences are with respect to the same variable, the construction is called a canonical vine (C-vine). If all mutual dependences are considered one after the other, i.e. the first with the second one, the third with the fourth one, etc., this is called a D-vine. C- and D-vines are special cases of regular vines, the latter being all possible pairwise decompositions. In the 3-D case there is no difference between a C- or a D-vine; only the ordering of variables can be changed.

Figure 1 illustrates the construction of a 3-D vine copula. In the first tree, three variables $-U, V, W-$ are given, and their pairwise dependences are captured by the bivariate copulas $C_{\mathrm{UV}}$ and $C_{\mathrm{VW}}$. These bivariate copulas can be conditioned under the variable $V$ through partial differentiation (Aas et al., 2009). This conditioning is indicated by dashed arrows in Fig. 1 and results in the conditional cumulative distribution functions $F_{\mathrm{U} \mid \mathrm{V}}$ and $F_{\mathrm{W} \mid \mathrm{V}}$ (see Eq. 1).

$$
F_{\mathrm{U} \mid \mathrm{V}}(u \mid v)=\frac{\partial C_{\mathrm{UV}}(u, v)}{\partial v}, \quad F_{\mathrm{W} \mid \mathrm{V}}(w \mid v)=\frac{\partial C_{\mathrm{VW}}(v, w)}{\partial v}
$$

In the second tree, the conditional CDF values are calculated for all triplets $(u, v, w)$ in the sample. These conditioned observations, which are again approximately uniformly distributed on $[0,1]$, are then used to fit another bivariate copula $C_{\mathrm{Uw} \mid \mathrm{V}}$. The full density function $c_{\mathrm{UVW}}$ of the 3-D copula is thus given by

$$
\begin{aligned}
c_{\mathrm{UVW}}(u, v, w)= & c_{\mathrm{UW} \mid \mathrm{V}}\left(F_{\mathrm{U} \mid \mathrm{V}}(u \mid v), F_{\mathrm{W} \mid \mathrm{V}}(w \mid v)\right) \\
& \cdot c_{\mathrm{UV}}(u, v) \cdot c_{\mathrm{VW}}(v, w) .
\end{aligned}
$$

It should be noted that the choice of the conditioning variable (i.e. $V$ ) is not unique, and different choices might lead to different results. In general, different vine copula decompositions differently approximate the underlying multivariate distribution (Hobæk Haff et al., 2010). In this paper the ordering of variables is based on the two bivariate copulas, $C_{\mathrm{UV}}$ and $C_{\mathrm{VW}}$, that fitted best considering the investigated copula families. The bivariate marginal distribution of $C_{\mathrm{UW}}$ is only implicitly modelled through the conditional joint distribution.

Thus, in order to derive the building blocks of the 3-D copula, three bivariate copulas $-C_{\mathrm{UV}}, C_{\mathrm{VW}}$ and $C_{\mathrm{UW} \mid \mathrm{V}}$ - need to be fitted. This is done stagewise and one can choose any of the available methods in the literature. Here, each bivariate copula is fitted by means of the maximum likelihood method, considering different copula families. The best fit is determined by the highest log-likelihood value (see Sect. 5.3).

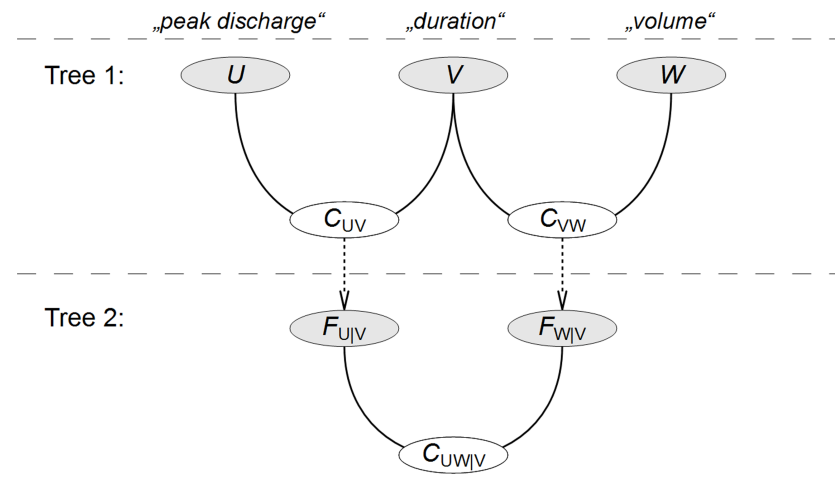

Fig. 1. Hierarchical nesting of bivariate copulas in the construction of a 3-D vine copula.

Several goodness-of-fit tests can be considered to validate the fitted bivariate copulas. In this paper the chosen goodness-of-fit test is the $A 7$ approach appearing in Berg (2009) and originating from Panchenko (2005). The advantage of this approach is that it estimates the distance between the two multivariate distribution functions without the need of any explicit dimension reduction, i.e. it is directly based on a comparison of observed pseudo-observations and simulated pseudo-observations under the null hypothesis. A simulation approach is taken to obtain the distribution of this test statistic under the null hypothesis. The original procedure as proposed by Berg (2009) is slightly altered in this paper as the test statistic of the hypothesis is averaged over the same number of simulations that are conducted during the simulation. A $p$ value estimate is derived from the fraction of test statistics exceeding this mean test statistic.

Combining the bivariate copulas as in Eq. (2) and substituting the marginal distribution functions $F_{X}, F_{Y}$ and $F_{Z}$ yields the 3-D distribution function of $(X, Y, Z)$. Let $f_{X}$, $f_{Y}$ and $f_{Z}$ denote the marginal density functions and define $u:=F_{X}^{-1}(x), v:=F_{Y}^{-1}(y)$ and $w:=F_{Z}^{-1}(z)$. The full density function $f_{X Y Z}$ of the distribution for any triplet $(x, y, z)$ is then given by

$$
\begin{aligned}
f_{X Y Z}(x, y, z):= & c_{\mathrm{UW} \mid \mathrm{V}}\left(F_{\mathrm{U} \mid \mathrm{V}}(u \mid v), F_{\mathrm{W} \mid \mathrm{V}}(w \mid v)\right) \\
& \cdot c_{\mathrm{UV}}(u, v) \cdot c_{\mathrm{VW}}(v, w) \\
& \cdot f_{X}(x) \cdot f_{Y}(y) \cdot f_{Z}(z) .
\end{aligned}
$$

The estimations in this paper have been done using $\mathrm{R}(\mathrm{R}$ Core Team, 2012), a free software environment for statistical computing, and the package spcopula ${ }^{2}$ building on the packages copula (Kojadinovic and Yan, 2010) and CDVine (Brechmann and Schepsmeier, 2011). The R scripts are available upon request from the authors. A demo related to this paper is available in the spcopula package.

\footnotetext{
${ }^{2}$ under development, available at R-Forge: http://r-forge. r-project.org/projects/spcopula
} 


\section{Estimating design events: definitions and methods}

In the literature and in practice, several approaches exist for estimating multivariate design events for a given design return period. The following sections provide a short overview of the most popular approaches, focusing on how a multivariate design event for a given return period could be calculated. In the specific case of multivariate joint return periods (JRP), typically a set of possible design events is found. In order to be able to assess the differences among the described approaches, we select the most probable of all possible design events. An ensemble-based design approach, in contrast to a single design event, will also be presented.

It is important to note that we present different classes of approaches: univariate (Sects. 3.1 and 3.2), bivariate (Sects. 3.3 and 3.4.1) and trivariate approaches (Sect. 3.4.2). In all cases, multivariate design events are provided; however, in the first case the procedure is based on the concept of a univariate return period, while in the second and third case the procedure is based on the concept of a bivariate and trivariate joint return period, respectively. This premise is pivotal since statistically these classes are incomparable due to the different intrinsic nature of the return period concepts. However, it is important to illustrate the differences in design events that stem from these modelling choices.

\subsection{Design events derived from a regression analysis}

A first approach is based on a univariate frequency analysis (denoted by REG). First, the driving variable $X$, i.e. the variable with a prominent role in the design, is chosen. Then a design return period $T_{\mathrm{REG}}$ is fixed, and given the marginal cumulative distribution of the design variable $F_{X}(x)$, the corresponding design quantile $x_{\text {REG }}$ (equal to the design quantile of the univariate approach $x_{\mathrm{UNI}}$ ) is sought, based on Eq. (3), with $\mu_{T}$ the mean interarrival time (typically given in years). In the case of annual maxima, $\mu_{T}$ equals $1 \mathrm{yr}$. Then, based on a linear regression of $X$ with the other design variable $Y$, the second design value $y_{\text {REG }}$ is obtained. This approach has been applied, among others, by Serinaldi and Grimaldi (2011):

$T_{\mathrm{REG}}=\frac{\mu_{T}}{1-F_{X}\left(x_{\mathrm{REG}}\right)} \Leftrightarrow x_{\mathrm{REG}}=F_{X}^{-1}\left(1-\frac{\mu_{T}}{T_{\mathrm{REG}}}\right)$

and some regression function $f_{\mathrm{REG}}$ modelling $Y$ in terms of $X$. Thus, $y_{\mathrm{REG}}:=f_{\mathrm{REG}}\left(x_{\mathrm{REG}}\right)$ is the predicted value based on the regression model for a given quantile $x_{\text {REG }}$ of the independent variable $X$. As previously mentioned, this approach does not provide an estimate following a joint return period definition. The motivation behind this approach is to provide a simple but statistically sound method when one can select a dominant driving variable in the practical application and only a small data set is available, hindering a deeper analysis.

\subsection{Design events derived from a bivariate conditional distribution}

A second approach (denoted by MAR) consists of conditioning the bivariate cumulative distribution function (CDF) $F_{X Y}(x, y)$ on the univariate marginal design quantile $x_{\mathrm{MAR}}=x_{\mathrm{UNI}}$ corresponding to the chosen univariate design return period $T_{\mathrm{UNI}}$. The resulting (univariate) conditional $\operatorname{CDF} F_{Y \mid X}\left(y \mid x=x_{\mathrm{UNI}}\right)$ can then be used to calculate the value $y_{\text {MAR }}$ for the conditional univariate design return period $T_{\mathrm{MAR}}$.

Advantage will be taken of the bivariate copula $C_{\mathrm{UV}}(u, v)$ to perform the calculation. With $u_{\mathrm{MAR}}=F_{X}\left(x_{\mathrm{MAR}}\right)$ and $v_{\mathrm{MAR}}=F_{Y}\left(y_{\mathrm{MAR}}\right)$, the procedure can be expressed as follows. We can rewrite the initial definition $T_{\mathrm{MAR}}=\frac{\mu_{T}}{1-F_{Y \mid X}\left(y \mid x=x_{\mathrm{MAR}}\right)}$ in terms of a copula with $U:=F_{X}(X)$ and $V:=F_{Y}(Y)$ as

$$
\begin{aligned}
T_{\mathrm{MAR}} & =\frac{\mu_{T}}{1-\left.\frac{\partial C_{\mathrm{UV}}\left(u, v_{\mathrm{MAR}}\right)}{\partial u}\right|_{u_{\mathrm{MAR}}:=1-\frac{\mu_{T}}{T_{\mathrm{UNI}}}}} \\
& =\frac{\mu_{T}}{1-C_{\mathrm{V} \mid \mathrm{U}=u_{\mathrm{MAR}}}\left(v_{\mathrm{MAR}}\right)} \\
\Leftrightarrow v_{\mathrm{MAR}} & =C_{\mathrm{V} \mid \mathrm{U}=u_{\mathrm{MAR}}}^{-1}\left(1-\frac{\mu_{T}}{T_{\mathrm{MAR}}}\right) .
\end{aligned}
$$

Inverse transformation yields

$y_{\mathrm{MAR}}=F_{Y}^{-1}\left(v_{\mathrm{MAR}}\right)$.

It should be noted that this approach does not result in a real bivariate design event having a joint return period in the strict sense as well as the afore-described regression based approach. The bivariate distribution is conditioned for the quantile of interest to the practitioner (corresponding with a univariate return period). This conditioned distribution is then used to obtain the other quantile, again based on the principles of a univariate return period. Therefore, the two obtained design quantiles $x_{\mathrm{MAR}}$ and $y_{\mathrm{MAR}}$ should not be considered as a real joint design event. Furthermore, one should keep in mind that the regression approach predicts the expected value for $Y$ given a certain quantile of $X$, while the conditional approach estimates the quantile of $Y$ conditioned under the quantile of $X$. Thus, both approaches cannot directly be compared from a probabilistic point of view, but are commonly found in the literature and are therefore included.

\subsection{Design events derived from a bivariate joint distribution}

Instead of using a conditional CDF, a widely used approach to calculate a bivariate return period can be followed which exploits the full bivariate $\mathrm{CDF} F_{X Y}(x, y)$. This can easily be expressed by means of a bivariate copula $C_{\mathrm{UV}}(u, v)$ with $U:=F_{X}(X)$ and $V:=F_{Y}(Y)$ as before. We refer to this approach as $\mathrm{OR}$ as it corresponds to the probability 
of $P[X>x \vee Y>y]$ following the notation introduced by Vandenberghe et al. (2011):

$$
\begin{aligned}
T_{\mathrm{OR}} & =\frac{\mu_{T}}{1-F_{X Y}\left(x_{\mathrm{OR}}, y_{\mathrm{OR}}\right)} \\
& =\frac{\mu_{T}}{1-C_{\mathrm{UV}}\left(F_{X}\left(x_{\mathrm{OR}}\right), F_{Y}\left(y_{\mathrm{OR}}\right)\right)} \\
& =\frac{\mu_{T}}{1-C_{\mathrm{UV}}\left(u_{\mathrm{OR}}, v_{\mathrm{OR}}\right)} .
\end{aligned}
$$

This approach is in fact an intuitive extension of the definition of a univariate return period. All couples $(u, v)$ that are at the same probability level $t_{\mathrm{OR}}=C_{\mathrm{UV}}(u, v)$ of the copula will have the same bivariate return period $T_{\mathrm{OR}}$. For a given design return period, the corresponding level $t_{\mathrm{OR}}$ can easily be calculated, the most likely design point $\left(u_{\mathrm{OR}}, v_{\mathrm{OR}}\right)$ of all possible events at this level can be obtained by selecting the point with the largest joint probability density:

$\left(u_{\mathrm{OR}}, v_{\mathrm{OR}}\right)=\underset{C_{\mathrm{UV}}(u, v)=t_{\mathrm{OR}}}{\operatorname{argmax}} f_{X Y}\left(F_{X}^{-1}(u), F_{Y}^{-1}(v)\right)$.

The corresponding design values $x_{\mathrm{OR}}$ and $y_{\mathrm{OR}}$ are easily calculated through the inverse CDFs:

$x_{\mathrm{OR}}=F_{X}^{-1}\left(u_{\mathrm{OR}}\right) \quad$ and $\quad y_{\mathrm{OR}}=F_{Y}^{-1}\left(v_{\mathrm{OR}}\right)$.

Once the joint density along the level curve is derived, one may consider different alternative approaches. Instead of the most likely event, one may calculate the expected value of the conditional distribution or calculate quantiles for given probabilities that might lead to a design approach incorporating more than a single design event. To limit the number of approaches, we will focus on the most likely event only, as e.g. used by Salvadori and De Michele (2012).

\subsection{Design events derived from a copula's Kendall distribution function}

Another definition of the bivariate return period is given by Salvadori and De Michele (2004); Salvadori (2004) and Salvadori et al. (2007). Recently, the concept of this bivariate secondary return period was extended to a complete multidimensional setting by Salvadori et al. (2011), called Kendall return period (denoted by KEN). This return period corresponds to the mean interarrival time of events more critical than the design event, the so-called super-critical or dangerous events. The super-critical events are potential threats to the structure and will appear more rarely than the given design return period. This partitioning of the probability distribution into a super-critical and non-critical region is based on the Kendall distribution function $K_{\mathrm{C}}$. This function is a univariate representation of multivariate information as it is the CDF of the copula's level curves: $K_{\mathrm{C}}(t)=P[C(u, v) \leq t]$. It allows for the calculation of the probability that a random point $(u, v)$ in the unit square has a smaller (or larger) copula value than a given critical probability level $t_{\mathrm{KEN}}$. The ability of the Kendall function to project a multidimensional distribution to a univariate one is similarly exploited by Kao and Govindaraju (2010) in the context of a joint deficit index for droughts.

The use of the Kendall distribution function to define the probability measure for calculating a JRP is advocated by Salvadori et al. (2011) as it is a theoretically sound multivariate approach sharing the notion of a critical layer, defined through the cumulative distribution function, with the univariate approach. The definition of the return period in both the univariate and in the multivariate Kendall approach is characterized by making a distinction between super-critical and non-critical events based on a critical cumulative probability level. The only way to extend this to a multivariate context is by using the Kendall distribution function. Probability measures that are constructed differently always entail events that will have a joint cumulative distribution function value that is larger or smaller than the critical probability level, and thus fail in subdividing the space between super-critical and non-critical events with respect to the joint cumulative distribution function. Following this avenue, any critical probability level $t_{\mathrm{KEN}}$ uniquely corresponds to a subdivision of the space into super-critical and non-critical regions. This is different from the OR case mentioned before, where in general different choices of critical events from the same critical probability level $t_{\mathrm{OR}}$ subdivide the space differently. From a return period point of view, the copula approach refers to super-critical events where at least one of the margins is larger than the design event, but the joint cumulative probability may be lower than the designated level yielding a shorter return period. On the other hand, the Kendallbased approach ensures that all super-critical events have a longer return period than the limit value, while some noncritical events might have larger marginal values than any selected design event.

For any given copula of any dimension, the Kendall distribution function can be calculated either analytically (e.g. for Archimedean copulas) or estimated numerically, and can thus be used to calculate the Kendall joint return period. Until now, only a very limited number of studies actually applied this kind of return period (e.g. Vandenberghe et al., 2010). In the following sections, the procedure for the 2-D and 3-D cases is outlined.

\subsubsection{2-D Kendall joint return period}

After choosing the design return period $T_{\mathrm{KEN} 2}$, the corresponding probability level $t_{\mathrm{KEN} 2}$ of the copula can be calculated by means of the inverse of the 2-D Kendall distribution function (Eq. 5). In 2-D this corresponds to finding an isoline on the copula. 


$$
\begin{aligned}
T_{\mathrm{KEN} 2} & =\frac{\mu_{T}}{1-K_{\mathrm{C}}\left(t_{\mathrm{KEN} 2}\right)} \\
\Leftrightarrow K_{\mathrm{C}}\left(t_{\mathrm{KEN} 2}\right) & =1-\frac{\mu_{T}}{T_{\mathrm{KEN} 2}} \\
\Leftrightarrow \quad t_{\mathrm{KEN} 2} & =K_{\mathrm{C}}^{-1}\left(1-\frac{\mu_{T}}{T_{\mathrm{KEN} 2}}\right) .
\end{aligned}
$$

When no analytical expression for $K_{\mathrm{C}}$ is available, the inverse can be calculated numerically based on an extensive simulation algorithm, described in Salvadori et al. (2011). Once $t_{\mathrm{KEN} 2}$ is known, the most likely design event in the unit square $\left(u_{\mathrm{KEN} 2}, v_{\mathrm{KEN} 2}\right)$ is selected on the corresponding isoline in an analogous way as described by Eq. (4). Through the use of the inverse of the marginal CDFs, the corresponding design event $\left(x_{\mathrm{KEN} 2}, y_{\mathrm{KEN} 2}\right)$ is found.

\subsubsection{3-D Kendall joint return period}

In three dimensions the corresponding probability level $t_{\mathrm{KEN} 3}$ should be found again in the same way as in Eq. (5). To calculate the inverse of the function $K_{\mathrm{C}}$, one might need to rely on a numerical method as, for instance, described by Salvadori et al. (2011). However, in contrast to the 2$\mathrm{D}$ case, the probability level $t_{\mathrm{KEN} 3}$ corresponds to an isosurface, i.e. all triplets $(u, v, w)$ on this surface have the same copula value $t_{\mathrm{KEN} 3}$. Generally, for a $n$-dimensional copula a isohypersurface of dimension $n-1$ exists that contains all $n$-dimensional points with the same copula level $t_{\mathrm{KENn}}$. A single design event $\left(u_{\mathrm{KEN} 3}, v_{\mathrm{KEN} 3}, w_{\mathrm{KEN} 3}\right)$ should again be selected on this isosurface. Therefore, the point $\left(u_{\mathrm{KEN} 3}, v_{\mathrm{KEN} 3}, w_{\mathrm{KEN} 3}\right)$ with the highest joint likelihood is selected, yielding the most likely event. In fact, this is the 3-D extension of the approach given in Eq. (4), i.e.:

$$
=\underset{C_{\mathrm{UVW}}(u, v, w)=t_{\mathrm{KEN} 3}}{\operatorname{argmax}} f_{X Y Z}\left(F_{X}^{-1}(u), F_{Y}^{-1}(v), F_{Z}^{-1}(w)\right) \text {. ( }
$$

\subsection{Theoretical comparison of JRP definitions}

The above-defined JRPs ( $T_{\mathrm{OR}}$ and $\left.T_{\mathrm{KEN}}\right)$ do not provide answers to the same problem statement. Therefore, one has to carefully consider the practical implications of the selected approach on the probability of interest. Vandenberghe et al. (2011) mentioned the inequality $T_{\mathrm{OR}} \leq T_{\mathrm{AND}}$, which can be extended to

$T_{\mathrm{OR}} \leq T_{\mathrm{KEN}} \leq T_{\mathrm{AND}}$

where $T_{\mathrm{AND}}$ refers to the exceedance probability of $P[X>x \wedge Y>y]$. The OR, AND and KEN JRPs can, in terms of 2-D copulas, be graphically interpreted on the unit square. The different return periods $T_{\mathrm{OR}}, T_{\mathrm{KEN} 2}$ and $T_{\mathrm{AND}}$ for a fixed design event $(u, v)$ can then, in every case, be expressed by $1 /(1-$ area(safe events)). This is shown in Fig. 2, where the areas represented by the different approaches for

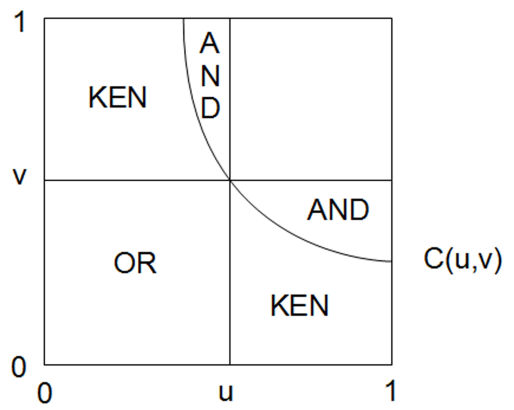

Fig. 2. Graphical representation of the different JRP definitions in terms of a copula (2-D case).

a given design event $(u, v)$ are indicated alongside with the copula level curve $C(u, v)$. It can be seen that the OR definition only declares all events in the lower-left rectangle as safe. The KEN approach declares the top-left and lower-right curved areas $(K E N)$ as safe as well, and they are added to the lower-left rectangle, yielding a larger return period for the same design event $(u, v)$. Lastly, the AND case adds the topleft and lower-right rectangles, resulting in the largest return period. Note that these inequalities hold only within the same dimensionality of a problem.

\subsection{Ensembles of design events}

From Secs. 3.3 and 3.4 it should be clear that for a design event characterized by several variables, one has to select an event out of a range of events which all share the same JRP. The selection of merely one event sensibly reduces the amount of information that can be obtained by the multivariate approach chosen. Volpi and Fiori (2012) present an approach to select a subset of the critical level to reflect the variability within the set of critical events. We follow a similar path and define a conditional distribution along the level curve to obtain a sample of the possible design events. The importance of an ensemble-based approach has already been stressed by Salvadori et al. (2011). Vandenberghe et al. (2010) provided a first attempt to benefit from the richness of an ensemble of critical values in a practical context.

Consider first the bivariate case, in which the JRP approaches based on copulas (OR, Sect. 3.3) and based on the Kendall distribution function (KEN2, Sect. 3.4.1) result in the finding of a contour level $t_{\mathrm{OR}}$ and $t_{\mathrm{KEN} 2}$ on which all pairs $(u, v)$ have the same respective JRP. Instead of using Eq. (4) to select the most likely point, the function $f_{X Y}$ over the $t$ isoline could be used as a univariate weight function out of which an ensemble of pairs could be sampled. In general, a rescaling is necessary to ensure that $f_{X Y}$ integrates to 1 and yields a probability density function (PDF) (Salvadori et al., 2011). Generally, not all pairs $(u, v)$ on the $t$ isoline have the same likelihood, i.e. pairs on the edges are less likely than pairs closer to the centre of the isoline. In this way, sampling according to $f_{X Y}$ makes more sense from a practical point 
of view than uniformly sampling over the isoline (as done by Vandenberghe et al., 2010).

Eventually, one will end up with an ensemble of pairs $\left(u_{i}, v_{i}\right)$ with $i$ ranging from 1 to $N$, the ensemble size. By means of the inverse marginal CDFs, these pairs are easily transformed to real values. This ensemble could then be used to run simulations from which the variability of specific design variables (e.g. thickness or height of a dam) can be assessed. This approach needs additional analysis as it will yield several design vectors going beyond the standard notion of a single design event. As an example, one could route an ensemble of 1000 pairs of peak discharge and volume through a dam model and consider the water height in the reservoir. Using just one design event only one water height is obtained. However, using the ensemble, information on the range and likelihood of possible water heights for the given design return period is obtained, making it possible to incorporate the variability within the design variables stemming from multiple design events along the critical level.

In the trivariate case (see Sect. 3.4.2) no isoline is obtained but an isosurface. Similar to the 2-D case, the full weight function over this isosurface could be rescaled to a bivariate probability density function out of which an ensemble of triplets could be sampled. The higher the dimensionality of the design problem, the more advantageous the ensemble approach becomes: in three dimensions more information is lost than in two dimensions by selecting just one design event. The drawback of the ensemble approach is the increasing need for run time when higher dimensions are considered.

\section{Differences among multivariate design events in the synthetic design hydrograph application}

\subsection{Experimental set-up}

In order to illustrate differences among estimated design events by the approaches described in the previous sections, a simulation experiment is set up and analysed with respect to the synthetic design hydrograph (SDH) attributes. The SDH is defined as a hydrograph with an assigned return period (uni- or multivariate), which can be characterized by random variables such as the peak discharge $Q_{\mathrm{p}}$, the duration $D$ and the volume $V_{\mathrm{p}}$. Specifically, given an observed or simulated run-off time series from which a set of extreme hydrographs is selected, one can determine the SDH shape in several ways (see Serinaldi and Grimaldi, 2011 and references therein). In a 2-D set-up, two hydrograph parameters (peak discharge and volume, peak discharge and duration or volume and duration) should be fixed, while the third one is obtained from the chosen hydrograph shape distribution. In a 3-D set-up the three characteristic parameters are obtained jointly.

In most common hydrological applications the interest is in the peak discharge $\left(Q_{\mathrm{p}}\right)$ and volume $\left(V_{\mathrm{p}}\right)$. Consequently, the 2-D analyses in this paper focus on these variables. However, as described in Sect. 3, there are several approaches that lead to the design values for $Q_{\mathrm{p}}$ and $V_{\mathrm{p}}$, including a 3-D approach. Applying the proposed approaches to the same data set allows comparison of the different underlying definitions and implications of the model selection. However, in a practical context one is typically tied to a specific frequency analysis that corresponds to the unique design characteristics.

The case study proposed in this paper consists of applying a continuous simulation model on a small, ungauged basin for which $500 \mathrm{yr}$ of synthetic direct run-off time series at a $5 \mathrm{~min}$ resolution are simulated. From this series the 500 maximum annual peaks are selected together with their corresponding hydrograph (identified as the continuous sequence of non-zero direct discharge values including the annual peak). Note that as direct discharge is considered, a zero discharge value does not imply a dry river. Consequently, $500\left(Q_{\mathrm{p}}, D, V_{\mathrm{p}}\right)$ triplets are available to which the described approaches estimating design events are applied. By considering a real case study, the obtained differences and hence the implications of a modelling choice can be evaluated in a practical context. In order to simulate the $500 \mathrm{yr}$ run-off time series, the COSMO4SUB model, described in the following section, is applied.

\subsection{The COSMO4SUB framework}

The synthetic data set on which the previously described approaches are applied is obtained through the use of the COSMO4SUB framework (Grimaldi et al., 2012d,c). COSMO4SUB is a continuous model which allows the simulation of synthetic direct run-off time series using minimal input information from rainfall data and digital terrain support. Specifically, the watershed digital elevation model (DEM) with a standard resolution used in hydrological modelling, the soil use and type, daily (preferably at least $30 \mathrm{yr}$ long) and sub-daily (preferably at least $5 \mathrm{yr}$ long) rainfall observations are the only data necessary to run the model. COSMO4SUB includes three modules: a rainfall time series simulator, a rainfall excess scheme and a geomorphological rainfall-run-off model. Next, the general principles are explained and in Sect. 5.1 specific details of the calibration are presented.

The first module is based on a single-site copulabased daily rainfall generator (Serinaldi, 2009) and on the continuous-in-scale universal multifractal model (Schertzer and Lovejoy, 1987) for disaggregating the daily rainfall to the desired time scale (up to $5 \mathrm{~min}$ ). The parameters included in this first module (six for each month for the daily rainfall simulator and three for the disaggregation model) are calibrated on the basis of the available rainfall observations (at two different scales).

The second module is related to the rainfall excess step. A new mixed Green-Ampt Curve Number (CN4GA Curve Number for Green Ampt) procedure was recently proposed 
(Grimaldi et al., 2012b) and included in the present version of the COSMO4SUB framework. The key concept is to use the initial abstraction (i.e. all the losses due to initial saturation, filling terrain gaps, interception, etc.) and the total SCS$\mathrm{CN}$ excess rainfall volume to estimate the effective saturated hydraulic conductivity and the ponding time of the GreenAmpt model. Consequently, the CN4GA approach tries to appropriately distribute the volume estimated by the SCS$\mathrm{CN}$ method over time. This module is characterized by five parameters (specified in Sect. 5.1) which are empirically assigned using the soil use and soil type map information. In addition, the event separation time $\left(T_{\mathrm{S}}\right)$ is included in this module since the continuous implementation of the SCS$\mathrm{CN}$ method requires to fix a no-rain time interval for which the cumulative gross and excess precipitation can be reset to zero. As shown in Grimaldi et al. (2012d,c), this parameter has a limited influence on the final results, and the value can be arbitrarily assigned in the range of 12-36 h.

The third module allows a continuous convolution of the rainfall excess to be carried out for obtaining the direct run-off time series through an advanced version of the width function instantaneous unit hydrograph (WFIUH). The adopted model, named WFIUH-1par (Grimaldi et al., 2010, 2012a), identifies the watershed IUH through the topographic information, and needs only one parameter that can be quantified referring to the watershed concentration time $\left(T_{\mathrm{c}}\right)$, estimated using empirical equations. Following the application of the three described modules, a continuous run-off scenario is obtained from which maximum annual hydrographs in terms of their peak discharge are selected. It is important to note that the variables duration and volume in the selected triplets do not necessarily reflect annual maxima.

\section{Data and materials}

This study is based on simulated data and a statistical model is fitted to this data set. This way a data set of sufficient size to compare the various approaches presented in this paper is obtained.

\subsection{Model set-up}

In order to provide a realistic scenario that can be used to evaluate the previously described approaches, the COSMO4SUB model was applied on the Torbido River, a small tributary of the Tiber River located in central Italy (watershed area: $61.67 \mathrm{~km}^{2}$ ). Basin elevations range from 85 to $625 \mathrm{~m}$, the average slope is $22 \%$, and the maximum distance between divide and outlet is $25.8 \mathrm{~km}$. The watershed DEM at a $20 \mathrm{~m}$ spatial resolution was provided by the Italian Geographic Military Institute (IGMI, 2003), while land cover was extracted from the CORINE database (EEA, 2000).

Observed rainfall data, useful for calibrating the two-stage rainfall simulator parameters, are available from the Castel
Cellesi rain gauge station for a period of $49 \mathrm{yr}$ at a daily time scale, and for a period of $10 \mathrm{yr}$ at a $5 \mathrm{~min}$ resolution (Serinaldi, 2009, 2010). For a description and evaluation of the $500 \mathrm{yr}$ rainfall synthetic time series, we refer to Grimaldi et al. $(2012 c, d)$.

\subsection{Annual extreme discharge events}

Once the $500 \mathrm{yr}$ synthetic direct run-off time series is determined, as described in Sect. 4.1, the 500 maximum annual peak discharge events are selected and characterized by their peak discharge $Q_{\mathrm{p}}$, duration $D$, and volume $V_{\mathrm{p}}$. For only six years the model provides zero direct run-off, which is reasonable considering the limited size of the watershed. These values are excluded in the following analyses.

All approaches rely on the marginal distribution functions of $Q_{\mathrm{p}}, D$ and $V_{\mathrm{p}}$ that need to be fitted in the first place. As the peak discharge variable consists of annual extreme values selected from the simulated $500 \mathrm{yr}$ discharge series, and the other two variables are closely correlated (but not necessarily annual maxima), the fit of several extreme value distributions is considered, i.e. the exponential, the Weibull and the generalized extreme value (GEV) distribution functions. These distributions are, respectively, a one-, two- and three-parameter distribution, allowing for various degrees of model complexity. Furthermore, the GEV distribution generally encompasses three different distributions, namely the Fréchet, the (reversed) Weibull and Gumbel distributions either directly, or through a transformation, as in the case of the Weibull distribution which corresponds to a reversed Weibull distribution. These different distribution types each represent a different kind of tail behaviour, namely a light tail (Gumbel), a heavy tail (Fréchet) and a bounded upper tail (Weibull). These behaviours can be separated based on the shape parameter $\xi$ of the GEV. Furthermore, the Weibull distribution is fitted separately as well, as it only corresponds to a GEV distribution after transformation. Finally, most extreme value distributions are of the exponential type, and cannot deal with an offset, i.e. when the smallest value of the variable in the CDF is larger than zero. However, as a result of censoring the zeros, the smallest value of the variables tends to be significantly higher than zero, leading to poor fits of the CDF. Therefore, a location parameter has been introduced in the distributions to ensure a proper fit in the tails.

A first test to ascertain the appropriate distribution for the three marginal variables is to display the empirical CDFs together with the directly fitted distribution. This is shown in Fig. 3, in which only the upper tail of the CDF is shown, i.e. the interval $[0.80,1]$ as the focus is on the extremes. It can immediately be seen that not all the distributions fit these tails equally well. This is corroborated by the Akaike information criterion (AIC) computed for all different models, shown in Table 1, as well as the log-likelihood of each model (not shown). Based on these criteria and Fig. 3, we select the Weibull distribution for $Q_{\mathrm{p}}$ and the exponential 
Table 1. The values of the AIC for the various distributions of the respective variables.

\begin{tabular}{lrrr}
\hline & GEV & Exponential & Weibull \\
\hline$Q_{\mathrm{p}}$ & 5370 & 5360 & 5326 \\
$D$ & 2610 & 2646 & 2928 \\
$V_{\mathrm{p}}$ & 14641 & 14599 & 14601 \\
\hline
\end{tabular}
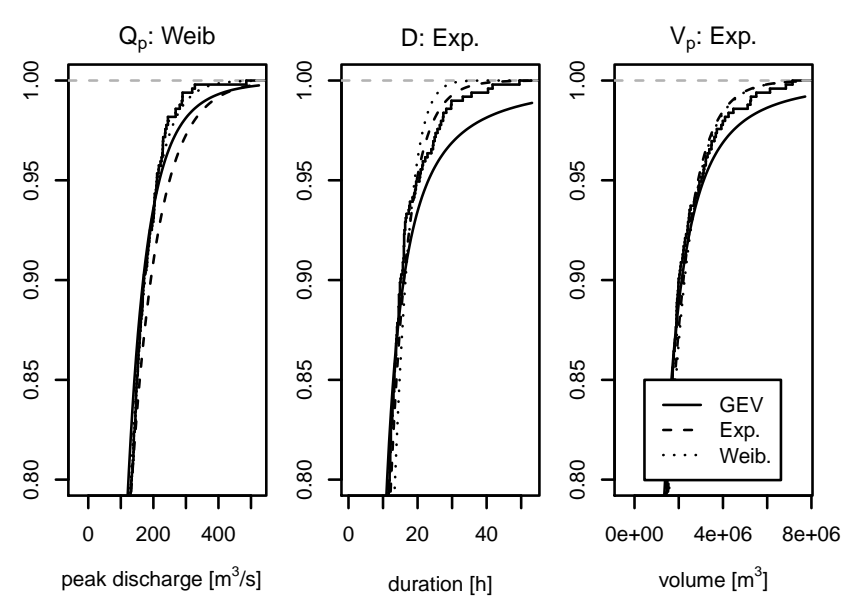

Fig. 3. The various cumulative distribution functions together with the empirical cumulative distribution function for the three variables. The best fitting distribution is denoted in the title of each graph.

distribution for $V_{\mathrm{p}}$. Seemingly, the GEV provides the overall best fit for $D$ according to the AIC, despite the poor representation of the upper tail (see Fig. 3). As the focal point of this study is set around a return period of ten years addressing the top $10 \%$ of the CDF, we chose to select the exponential distribution because of its better fit in this region. More indepth testing through Q-Q plots (not shown here) indicates that this is indeed a better approximation of the distribution. Further investigation of additional distribution families and combinations of these might improve the fit of the marginals, but is out of scope of this paper. Nevertheless, a good fit of the marginal distributions is key to the practical application. Hence, the following models are selected:

- $Q_{\mathrm{p}}$ : Weibull (Anderson-Darling $p=0.59$ ),

- $D$ : exponential (Anderson-Darling $p=0.08$ ),

- $V_{\mathrm{p}}$ : exponential (Anderson-Darling $p=0.18$ ).

Here, the Anderson-Darling test was used to determine whether the samples were significantly different from the fitted distributions. It should be understood that a consistency in marginal distribution functions across the different approaches is far more important for comparison reasons than a perfect fit, considering the underlying data are simulations.

To analyse the association between the variables, which will be modelled by means of copulas, Kendall's tau is

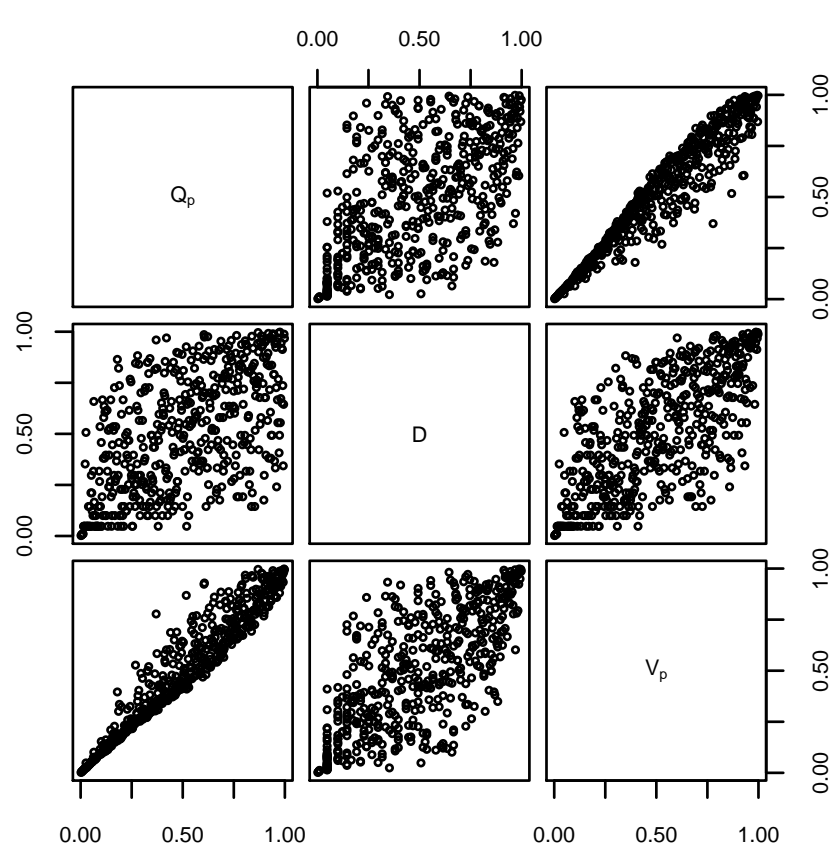

Fig. 4. Normalized rank scatter plots for all pairs of variables. Kendall's tau is 0.85 for $\left(Q_{\mathrm{p}}, V_{\mathrm{p}}\right), 0.42$ for $\left(Q_{\mathrm{p}}, D\right)$ and 0.54 for $\left(V_{\mathrm{p}}, D\right)$.

calculated, and normalized rank scatterplots are evaluated for each pair of variables (Fig. 4). Evidently, there are strong positive associations. Also, some ties are present, especially for $D$, which have been assigned with their mean rank in the transformation. The next section deals with the modelling of these associations.

\subsection{Fitting of the 2-D and 3-D copulas}

As described in Sect. 2.2, we used maximum likelihood estimation to fit a copula from each investigated family for every pair of variables and selected the best fitting one by the highest log-likelihood value. The copula families investigated include Gaussian, Student, Gumbel, Frank, Clayton, BB1, BB6, BB7, BB8 and the survival copulas of the 4 latter ones (details on all these families can be found in Nelsen (2006) and Joe (1997)). Table 2 gives an overview of the parameters and goodness-of-fit results. The $p$ values are estimated from 1000 iterations each.

The following approaches in the 2-D case make use of the fitted BB7 copula $C_{13}$ which models the dependence between $Q_{\mathrm{p}}$ and $V_{\mathrm{p}}$. It should be noted that this copula is not able to represent the boundary effect present in the rank scatter plot (Fig. 4). To the authors' knowledge, no copula is available in the literature that would be able to model such a boundary effect. As the BB7 copula family belongs to the class of Archimedean copulas, its Kendall distribution function can easily be obtained analytically.

For the $3-\mathrm{D}$ case, the three fitted bivariate copulas $C_{12}$, $C_{23}$ and $C_{13 \mid 2}$ are then composed into the $3-\mathrm{D}$ vine copula 
Table 2. An overview of the fitted bivariate copulas in the 2-D copula-based and 3-D vine copula-based approach.

\begin{tabular}{lllclccc}
\hline & Pairs of variables & ID & $\tau_{K}[-]$ & Copula family & Parameters & $p$ value \\
\hline 2-D & $Q_{\mathrm{p}} \sim V_{\mathrm{p}}$ & 13 & 0.85 & BB7 & 2.24 & 14.10 & 0.69 \\
\hline \multirow{2}{*}{ 3-D } & $Q_{\mathrm{p}} \sim D$ & 12 & 0.42 & survival BB7 & 2.05 & 0.35 & 0.74 \\
& $D \sim V_{\mathrm{p}}$ & 23 & 0.54 & survival BB7 & 2.25 & 1.09 & 0.75 \\
& $\left(Q_{\mathrm{p}} \sim V_{\mathrm{p}}\right) \mid D$ & $13 \mid 2$ & 0.83 & Student copula & 0.96 & 2.00 & 0.66 \\
\hline
\end{tabular}

as given in Eq. (2). For comparison purposes, 3-D copula fits for the three-parameter Gaussian copula and the oneparameter Clayton, Frank, and Gumbel copulas have also been performed. The log-likelihood shows a $10 \%$ increase for the fitted vine copula (1047) with respect to the Gaussian one (935), while the three one-parameter Archimedean copulas have far smaller values (432-532). Thus, the vine copula yields the best fit within this set of copula families in terms of the log-likelihood. As no closed form exists for the cumulative distribution function of this vine copula, a numerical evaluation based on a sample of 100000 points was carried out in order to be able to calculate the (inverse of the) Kendall distribution function.

However, the singularity appearing in Fig. 4 for the pair $\left(Q_{\mathrm{p}}, V_{\mathrm{p}}\right)$ is neglected in both, the bivariate and the vine copula (as well as in the other considered copulas). In the bivariate case, no copula family with such a limited support could be found, while the vine copula's decomposition is based on the bivariate copulas $C_{12}$ and $C_{23}$ addressing the pair $\left(Q_{\mathrm{p}}, V_{\mathrm{p}}\right)$ only through the conditional joint distribution. Thus, all investigated copula families would in general sample unrealistic point pairs $\left(Q_{\mathrm{p}}, V_{\mathrm{p}}\right)$ beyond the border appearing in the scatter plot. A discussion on this singularity and its underlying process can be found in Serinaldi (2013).

\section{Results and discussion}

\subsection{Calculation of single design events}

In this section the design values for the SDH with a design return period of $10 \mathrm{yr}$ are calculated based on the 2-D and 3 -D approaches presented in Sect. 3. The triplet $\left(Q_{\mathrm{p}}, D, V_{\mathrm{p}}\right)$ is considered for which the following transformations hold

$U=F_{Q_{\mathrm{p}}}\left(Q_{\mathrm{p}}\right), V=F_{\mathrm{D}}(D)$ and $W=F_{V_{\mathrm{p}}}\left(V_{\mathrm{p}}\right)$

As a reference the univariate case is analysed first. Based on the inverse of the CDFs $F_{Q_{\mathrm{p}}}, F_{\mathrm{D}}$ and $F_{V_{\mathrm{p}}}$, at a probability level of $1-\frac{\mu_{T}}{T_{\mathrm{UNI}}}=1-\frac{1}{10}=0.9$, the design values $q_{\mathrm{p}, \mathrm{UNI}}=174 \mathrm{~m}^{3} \mathrm{~s}^{-1}, v_{\mathrm{p}, \mathrm{UNI}}=2.21 \times 10^{6} \mathrm{~m}^{3}$ and $d_{\mathrm{UNI}}=16.02 \mathrm{~h}$ are obtained. In the following, Table 3 and Fig. 6 provide a way to compare these and all further estimated design events. In order to be able to compare design events with the data, the simulated pairs $\left(Q_{\mathrm{p}}, V_{\mathrm{p}}\right)$ are

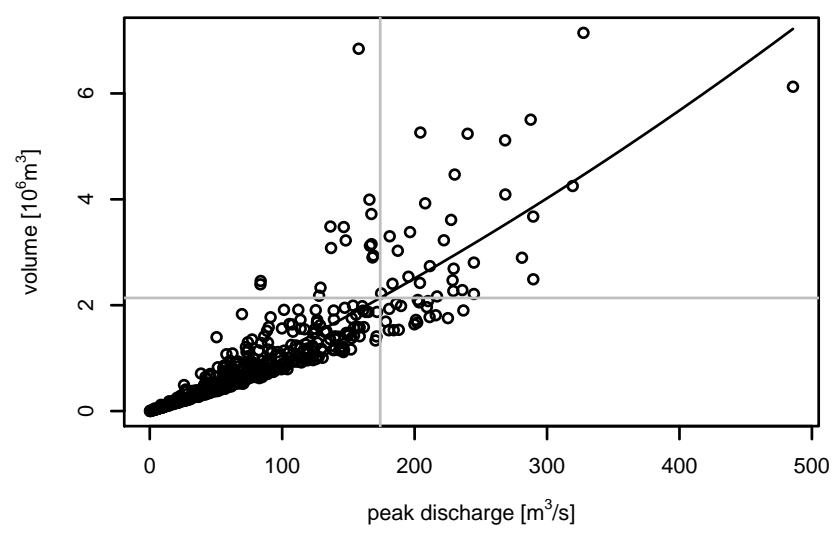

Fig. 5. Illustration of the derivation of the design quantiles based on the regression approach.

visualized as grey dots in Fig. 6 that summarizes all described approaches.

First the 2-D case is considered, in which the focus is on the couple $\left(Q_{\mathrm{p}}, V_{\mathrm{p}}\right)$. In the regression-based approach (REG, Sect. 3.1) the starting point is the univariately derived quantile $q_{\mathrm{p}, \mathrm{UNI}}$, being usually the driving variable in many hydrological applications (see Eq. 3). Based on a regression between $Q_{\mathrm{p}}$ and $V_{\mathrm{p}}$, as shown in Fig. 5, the design volume $v_{\mathrm{p}, \mathrm{REG}}$ is easily estimated as $2.14 \times 10^{6} \mathrm{~m}^{3}$. This volume is lower than the one obtained by a purely univariate analysis partly due to the different definition based on the expectation instead of a quantile.

The second 2-D approach is based on the conditional copula (MAR, Sect. 3.2). The conditioning of the bivariate copula $C_{\mathrm{UW}}$ (denoted as $C_{13}$ in Sect. 5.3) for $u_{\mathrm{UNI}}=0.9$ results in the function $C_{\mathrm{W} \mid \mathrm{U}}(w, u=0.9)$. The value of $w_{\mathrm{MAR}}=0.9521$ corresponds with a probability level of 0.9 . By means of the inverse $F_{V_{\mathrm{p}}}^{-1}\left(w_{\mathrm{MAR}}\right)$, the design volume $v_{\mathrm{p}, \mathrm{MAR}}$ is calculated as $2.92 \times 10^{6} \mathrm{~m}^{3}$, which is considerably larger than the former design volumes.

The true joint return period approaches based on the bivariate copula $C_{\mathrm{UW}}(\mathrm{OR}$, Sect. 3.3) is the third 2-D approach. For $T_{\mathrm{OR}}=10 \mathrm{yr}$, the corresponding copula level $t_{\mathrm{OR}}$ equals 0.9 , and corresponds to an isoline. Using the marginal CDFs for $Q_{\mathrm{p}}$ and $V_{\mathrm{p}}$, Eq. (4) can be solved to find the point $\left(u_{\mathrm{OR}}, w_{\mathrm{OR}}\right)$ with the highest joint likelihood, i.e. $\left(u_{\mathrm{OR}}, w_{\mathrm{OR}}\right)=(0.927,0.925)$. Using the inverse CDFs 
Table 3. Overview of the calculated design event for $T=10 \mathrm{yr}$, based on several approaches. The values are rounded to address the limited numerical precision and ease comparison.

\begin{tabular}{lllllllllc}
\hline Approach & Subscr. & $\begin{array}{l}K_{\mathrm{C}} \\
{[-]}\end{array}$ & $\begin{array}{l}u_{T} \\
{[-]}\end{array}$ & $\begin{array}{l}v_{T} \\
{[-]}\end{array}$ & $\begin{array}{l}w_{T} \\
{[-]}\end{array}$ & $\begin{array}{c}q_{\mathrm{p}, T} \\
{[-]}\end{array}$ & $\begin{array}{c}d_{T} \\
{\left[\mathrm{~m}^{3} \mathrm{~s}^{-1}\right]}\end{array}$ & $\begin{array}{c}v_{\mathrm{p}, T} \\
{\left[10^{6} \mathrm{~m}^{3}\right]}\end{array}$ \\
\hline univariate & UNI & $\times$ & $\times$ & 0.9 & 0.9 & 0.9 & 174 & 16.02 & 2.21 \\
lin. regr. & REG & $\times$ & $\times$ & 0.9 & $\times$ & 0.892 & 174 & $\times$ & 2.14 \\
cond. cop. & MAR & 0.9 & $\times$ & 0.9 & $\times$ & 0.952 & 174 & $\times$ & 2.92 \\
copula 2-D & OR & 0.9 & $\times$ & 0.927 & $\times$ & 0.925 & 192 & $\times$ & 2.49 \\
$K_{\mathrm{C}-2 D}$ & KEN2 & 0.836 & 0.9 & 0.877 & $\times$ & 0.875 & 161 & $\times$ & 1.99 \\
$K_{\mathrm{C}-3 D}$ & KEN3 & 0.730 & 0.9 & 0.844 & 0.820 & 0.851 & 147 & 12.90 & 1.83 \\
\hline
\end{tabular}

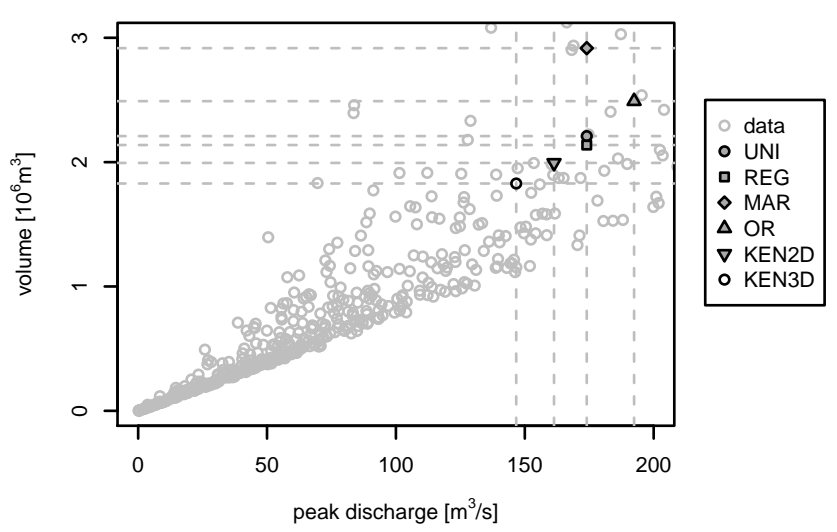

Fig. 6. An overview of the different design values for a design return period of $10 \mathrm{yr}$ obtained with the different definitions. Note that only a subset is shown and the data points exceed both axes.

the design event is obtained: $\left(q_{\mathrm{p}, \mathrm{OR}}, v_{\mathrm{p}, \mathrm{OR}}\right)=\left(192 \mathrm{~m}^{3} \mathrm{~s}^{-1}\right.$, $2.49 \times 10^{6} \mathrm{~m}^{3}$ ). Both the design peak discharge $Q_{\mathrm{p}}$ and the volume $V_{\mathrm{p}}$ are larger than what is obtained in the univariate case.

The last 2-D approach is the one in which the JRP is calculated using the Kendall distribution function (KEN2, Sect. 3.4.1). Here, the focus is on the inverse of $K_{\mathrm{C}}$ for a probability level of 0.9: $t_{\mathrm{KEN} 2}=K_{\mathrm{C}}^{-1}(0.9)$. The Kendall distribution function of the bivariate copula $C_{\mathrm{UW}}$, allows calculation of the $t_{\mathrm{KEN} 2}$ level corresponding to a cumulative probability of 0.9 , i.e. $t_{\mathrm{KEN} 2}=0.836$. This level is smaller than the one obtained in the former copula-based JRP approach. Again, Eq. (4) can be solved to obtain the most likely design event $\left(u_{\mathrm{KEN} 2}, w_{\mathrm{KEN} 2}\right)=(0.877,0.875)$. Transformation to the real domain by means of the inverse CDFs results in the design event $\left(q_{\mathrm{p}, \mathrm{KEN} 2}, v_{\mathrm{p}, \mathrm{KEN} 2}\right)=\left(161 \mathrm{~m}^{3} \mathrm{~s}^{-1}\right.$, $\left.1.99 \times 10^{6} \mathrm{~m}^{3}\right)$.

Besides the estimation of 2-D design events, also one approach for estimating a 3-D design event is presented in Sect. 3.4.2 together with the fitted 3-D vine copula (see Sect. 5.3). The 3-D vine copula is used for simulating 100000 triplets $(u, v, w)$ as a basis for the numerical inversion of the Kendall distribution function. Here, the probability level of 0.9 corresponds to a $t_{\mathrm{KEN} 3}$ level of 0.730 on the 3-D vine copula. In contrast to the 2-D approaches, the $t_{\mathrm{KEN} 3}$ level corresponds to a surface. Using the marginal CDFs in combination with Eq. (6), the most likely point on this surface is found as $\left(u_{\mathrm{KEN} 3}, v_{\mathrm{KEN} 3}, w_{\mathrm{KEN} 3}\right)=(0.844$, $0.820,0.851)$. Using the inverse CDFs this results in the design event $\left(q_{\mathrm{p}, \mathrm{KEN} 3}, d_{\mathrm{KEN} 3}, v_{\mathrm{p}, \mathrm{KEN} 3}\right)=\left(147 \mathrm{~m}^{3} \mathrm{~s}^{-1}, 12.90 \mathrm{~h}\right.$, $\left.1.83 \times 10^{6} \mathrm{~m}^{3}\right)$. Note that the Kendall distribution function is a univariate representation of multivariate information and that its form is different in the 2-D and 3-D cases.

\subsection{Obtaining an ensemble of design events}

The preceding analyses resulted in a single design event; however, as stated in Sect. 3.6, the generation of an ensemble would be preferable. For example, consider the approach where the JRP is based on the Kendall distribution function in the 2-D case. The $t_{\mathrm{KEN} 2}$ level was found to be 0.836 for a 2-D Kendall-based JRP of $10 \mathrm{yr}$ (see Table 3 ). Figure 7 shows this $t_{\mathrm{KEN} 2}$ level and the $t_{\mathrm{OR}}$ level of 0.9 , together with the earlier, identified most likely design events $\left(u_{\mathrm{KEN} 2}, w_{\mathrm{KEN} 2}\right)$ and $\left(u_{\mathrm{OR}}, w_{\mathrm{OR}}\right)$ along with a sample of size 500 each. Obviously, along this contour the occurrence of several other events is possible. The sampling across these contours according to the likelihood function results in ensembles of events all having a copula-based and 2-D Kendall-based JRP equal to $10 \mathrm{yr}$, respectively. All sampled events clearly lie on a contour, corresponding with the $t_{\mathrm{OR}}$ level and $t_{\mathrm{KEN} 2}$ level. According to the greyscale, the highest density of design events is sampled around the most likely realization, whereas less design events are sampled on the two outer limits of each contour.

The density of the ensembles across these contours could be projected (and normalized) on both the $Q_{\mathrm{p}}$ and $V_{\mathrm{p}}$ axis, resulting in univariate PDFs for $Q_{\mathrm{p}}$ and $V_{\mathrm{p}}$ underlying the ensembles. These are shown in Figs. 8 and 9. The most likely design events are naturally situated at the maximum of these PDFs. In general, these conditional distributions do not have to be bounded and extremely large events might possess a positive likelihood.

These PDFs hold a lot of information on the design events. For example, $90 \%$ of all design events with a 2-D Kendallbased JRP equal to $10 \mathrm{yr}$ have a peak discharge in the range 


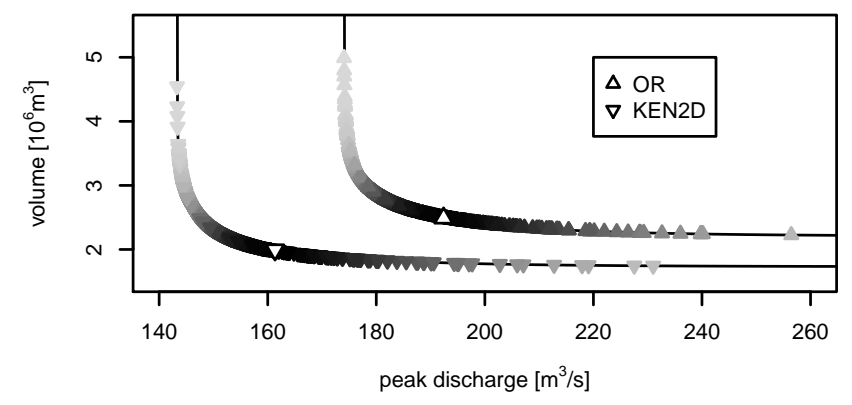

Fig. 7. An ensemble of $500\left(q_{\mathrm{p}}, v_{\mathrm{p}}\right)$ pairs that all have a copulabased and 2-D Kendall-based JRP of $10 \mathrm{yr}$, respectively. The density of the ensemble is given in greyscale: the brighter the grey, the less events sampled. The most likely event is also indicated.

of $\left[150 \mathrm{~m}^{3} \mathrm{~s}^{-1}, 238 \mathrm{~m}^{3} \mathrm{~s}^{-1}\right]$ and a volume in $\left[183 \times 10^{6} \mathrm{~m}^{3}\right.$, $326 \times 10^{6} \mathrm{~m}^{3}$ ]. Note from Fig. 7 that lower volumes occur together with higher peak discharge values and vice versa. As briefly mentioned in Sect. 3.6, the ensemble of design events can also be used to calculate another design variable, such as the water height in a reservoir, for which again a PDF of possible design values can easily be obtained. However, this exercise is beyond the scope of this paper.

\subsection{Uncertainty in the design event estimation}

In the previous section and Sect. 3.6, we discussed that for a given return period, different design events can be selected due to the multivariate nature of the design problem and that the designer can make use of this variability for parameterizing hydraulic structures. From the shown analysis, it is also clear that the presented approaches provide different design event estimates. Yet, these approaches are also prone to uncertainty because of the fact that the copula or the model used to select the design event is fitted to a (small) number of extreme events in an observed time series. Variations in the time series might lead to different model parameters and hence result in alternative design events. The question can thus be posed whether the different approaches generate statistically different design events if one accounts for the uncertainty due to fitting of the probabilistic model. To answer this question the uncertainty has to be addressed, resulting in confidence bands. As no closed form exists, a common approach is to run simulations. In each simulation step, we sampled 494 pairs (the same number as originally observed pairs) from our fitted bivariate distribution and re-estimated the copula and marginal parameters. From the newly obtained probabilistic model, all approaches provided the most likely design event estimate (resulting in the scattered estimates shown as squares and triangles in Fig. 10). For each approach, the 0.025 -quantile and 0.975 -quantile design events out of all simulated ones are selected in terms of their return period definition for the null-hypotheses model. These quantiles describe the border of the $95 \%$ confidence band

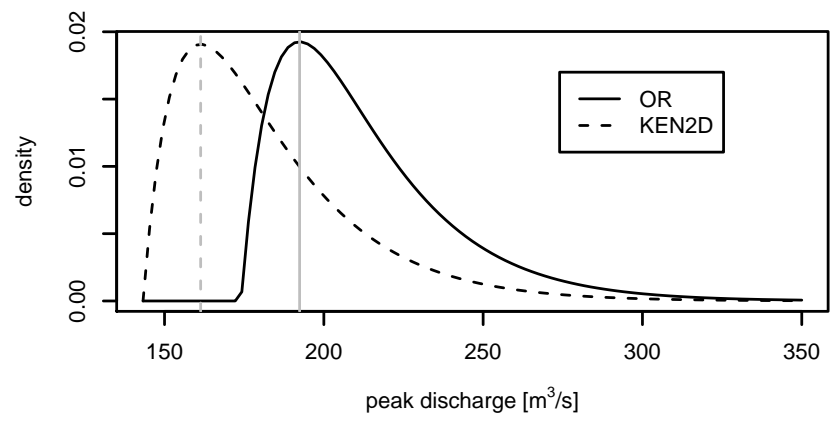

Fig. 8. PDF of $Q_{\mathrm{p}}$ in the OR and KEN2D ensembles. The most likely design discharge values are indicated by vertical lines.

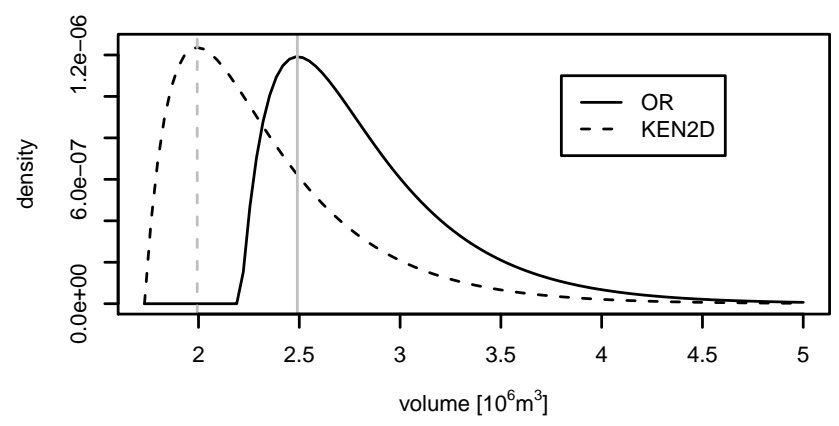

Fig. 9. PDF of $V_{\mathrm{p}}$ in the OR and KEN2D ensembles. The most likely design volumes are indicated by vertical lines.

denoted by the corresponding copula t-level curve. The results are summarized in Fig. 10. As the inner $95 \%$ of points of each cloud do not intersect between the three approaches, it is evident that the predicted design events are significantly different. However, projecting the multivariate design events to their univariate margins, all confidence intervals intersect except for the univariate predicted volume and the design volume based on the conditional copula (MAR). Addressing the additional uncertainty of the estimates due to the selection of merely a single design event, the dashed curved lines in Fig. 10 provide an approximation. They limit the region where $95 \%$ of the simulated t-level curves fall. Ensembles of design events would then be drawn along these level curves. Thus, the copula-based (OR) and the Kendallbased (KEN2D) approach provide significantly different design event ensembles.

\subsection{Some practical considerations}

Table 3, Figs. 6 and 10 clearly demonstrate that the choice of the estimation approach influences the design event values. This evidently is something the practitioner should consider when designing a hydraulic structure, e.g. a dam, based on a specific design hydrograph, as it directly influences the safety and the cost of the structure to be built.

With respect to the univariate design quantile $q_{\mathrm{p}, \mathrm{UNI}}$, only the approach using the copula-based JRP provides a larger 


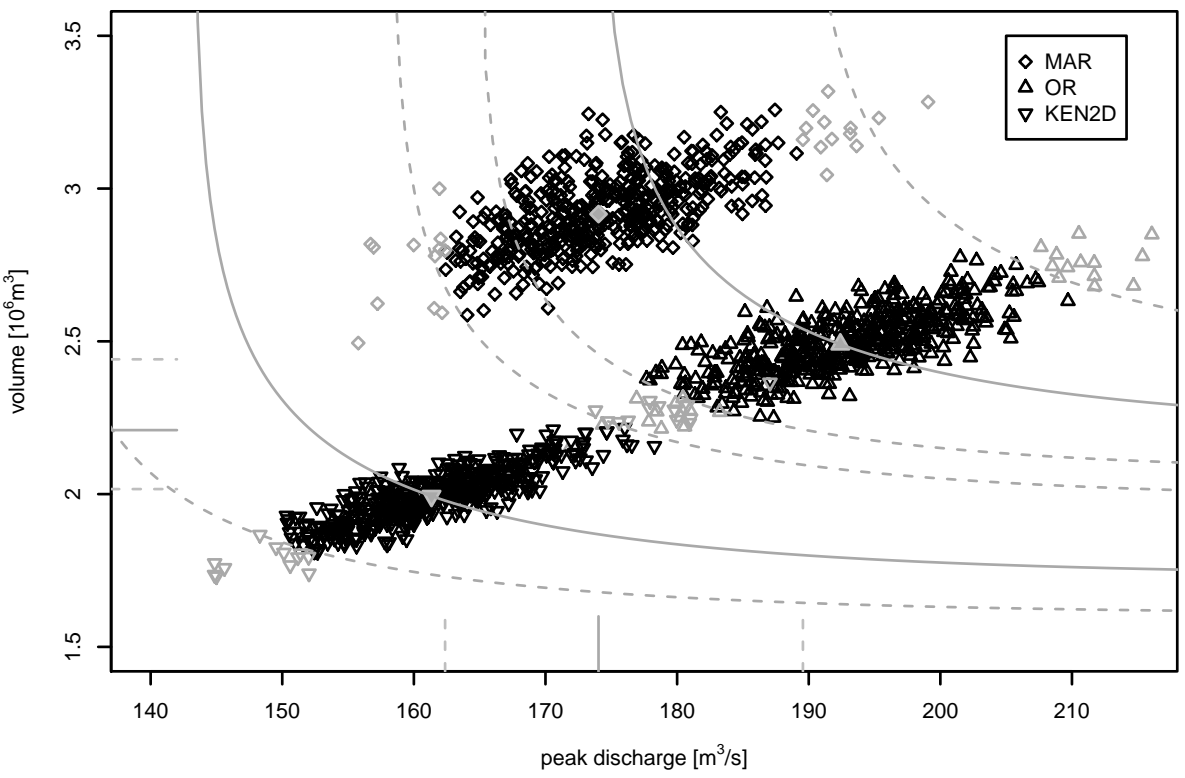

Fig. 10. 500 simulations of different approaches to obtain a single design event with a design return period of $10 \mathrm{yr}$. Straight line segments indicate the univariate approaches and curved lines represent the bivariate approaches, with dashed lines approximating the ensemble confidence band estimates for their corresponding point cloud each. The estimated design events are denoted as filled shapes. The open grey shapes represent the most and least extreme $2.5 \%$ for each approach.

quantile, whereas for the approaches using the Kendall-based JRP a smaller quantile is found. The other approaches use the univariate quantile as a starting point, resulting in identical quantiles. Considering the design quantiles $v_{\mathrm{p}, \text {, }}$, the MAR and OR approaches yield a larger quantile than the univariate quantile, while the REG and Kendall-based approaches yield smaller values.

The three true JRP approaches use information of the full bivariate (copula-based and 2-D Kendall-based approach) or trivariate (3-D Kendall-based approach) distribution function. The Kendall-based approaches have the advantage of using a mathematically consistent way of defining the probability of extremes or dangerous events relying on the CDF as in the univariate approach, unlike the JRP approach based on the copula solely. For a full discussion please refer to Salvadori et al. (2011). However, there is no universal choice of an appropriate approach to all real-world problems. The most important is to address the problem from a probabilistic point of view and to be aware of the practical implications of the approach chosen (outlined in this paper). It is also evident, but not necessarily the case, that the more variables are included (2-D vs. 3-D), the smaller the design quantiles become. Salvadori and De Michele (2012) discuss this dimensionality paradox and provide a theoretical explanation for it.

Furthermore, the issue of selecting just one design event out of a range of events all having the same joint return period (i.e. on an isoline or isosurface of the copula) could be seen as a drawback of the multivariate approaches available in the literature, as the most likely event does not necessarily correspond to the most severe one for a given hydraulic structure. However, there is the full potential to set a step aside from this one-event-design approach to a full ensemblebased design approach. Therefore, this paper includes an approach for the generation of a design ensemble. It is clear that the ensemble approach provides a lot more information on the possible outcome of design events. The proposed ensemble-based approach entails the most likely design event, but furthermore provides a clear idea on the probability that other events (but all having the same JRP) will occur. Checking these ensembles against the desired design of the hydraulic structure will illustrate the real threat to the structure. It therefore provides a way of assessing some uncertainty of the design variables associated with the selection of a single design event. If a single design event is sought, the pure copula-based approach (Sect. 3.3) has the advantage of guaranteeing that only a fraction of $1 / T_{\mathrm{OR}}$ events exceeds the margins of any of the possible estimated design events.

It should also be noted that the fitting of the copulas (bivariate, trivariate or multivariate) is a very important part of the design event estimation. If the practitioner is not acquainted with this initial aspect of design studies, it is very easy to make wrong choices. Naturally, the multidimensional approaches require a larger data set in order to produce robust parameter estimates. Thus, the length of the time series and the amount of missing data have to be considered before an approach is selected. The authors of this work believe that the vine copula approach is the way to go for constructing flexible multivariate distribution functions, as it enables use of more widely spread bivariate copulas as building blocks for 
more complex multivariate distribution functions. Of course, a good balance between the number of variables considered and the (numerical) complexity of the vine copula should be sought, keeping in mind that all this also affects the eventual design. Further studies are necessary to assess the sensitivity of the JRP analysis to sample size and sample selection.

In general, the Kendall-based approach can be applied to any copula and can be used for both large (e.g. floods) and small (e.g. droughts) extremes. However, one should also be aware of the fact that the approaches in this study are only applied to variables that are positively associated and with a focus on extremes in terms of large values. In all other cases, adaptations should be made in order to operate in the right area of the copula. Further applications of the copulabased and Kendall-based JRP approaches in other case studies should provide more insight on this in the near future. Serinaldi (2012) highlighted the potentially misleading notion of return periods, and suggests to report return periods alongside with annual exceedance probabilities as done e.g. by Theiling and Burant (2012). This aspect should be included in further studies as well.

\section{Conclusions}

The aim of this study was to provide an overview of stateof-the-art approaches to estimate design events for a given return period and to discuss their differences in a practical application. Therefore, a synthetic case study focusing on the estimation of design parameters for a synthetic design hydrograph (SDH) was considered. As they are the most important SDH variables, the peak discharge $Q_{\mathrm{p}}$, its duration $D$, and volume $V_{\mathrm{p}}$ were chosen.

In first instance a review of several approaches yielding design events available in the recent literature was provided focusing on how to apply these. As multiple variables were considered in the different return period approaches, an important aspect is (the modelling of) the dependence between variables. In this context the potential and the use of copulas for the construction of multivariate distribution functions was stressed and illustrated. On the one hand, a bivariate copula of $\left(Q_{\mathrm{p}}, V_{\mathrm{p}}\right)$ was fitted. On the other hand, also the fitting of the trivariate copula of $\left(Q_{\mathrm{p}}, D, V_{\mathrm{p}}\right)$ was elaborated in a comprehensive way by means of the vine copula approach.

Eventually, design events for a $10 \mathrm{yr}$ joint return period were obtained considering a 2-D regression-based, a 2-D conditional copula-based, a 2-D copula-based, a 2-D Kendall-based, and a 3-D Kendall-based approach. The traditional 1-D return period definition is considered as a reference for comparison purposes. Differences in design quantiles were discussed while also the theoretical appropriateness was explained. This paper warns practitioners against blind use of just one available design event estimation approach, and stresses the importance of good copula fitting and the effect on the eventual design event outcome. A simulation study showed that the investigated approaches yield statistically different design events. Thus, the predictions are not only different following the theoretical inequality (Eq. 7), but do withstand the variability due to uncertainties associated with the probabilistic model fitted to the data. Based on the available literature and the case study in this paper the copula-based and Kendall-based JRP approaches are valuable multivariate extensions of the univariate approaches. However, their applicability always depends on the availability of data and the probabilistic nature of the actual real-world problem. For constructing multivariate copulas, the vine copula method is advised.

Further (joint) research efforts should focus on a shift from one-design-event approaches to ensemble-design-event approaches, enabling incorporation of the variability in the design event selection. A first valuable approach to this ensemble-based design was provided in this paper. The ultimate goal should be the elaboration of a useful and understandable framework for multivariate frequency analyses, with clear guidelines to practitioners.

From a practical perspective, it is impossible to provide a general suggestion for an appropriate approach to estimate multivariate design events applicable to a vast set of design exercises. Firstly, as previously described, the available approaches are different from a statistical point of view. Until now many applications are based on the concept of univariate return periods, as the concept of multivariate return periods has a different meaning and is potentially less conservative. Secondly, the best approach, in our opinion, is related to the hydraulic structure to be designed. Different design exercises might be critical to single variables, which should then be selected as the driving component in the data selection and in the modelling process. If one is, for example, interested in the hydrograph volume for designing a reservoir for flood regulation, it is essential to understand whether there is a predominant driving variable. Specifically, if the reservoir is regulated by a levee, the volume design is related to a specific discharge value. In this case the bivariate conditional distribution could be preferred, since the discharge analysis is performed with a standard univariate approach and the volume return period is estimated conditioned on the discharge design value. In similar practical problems, the regression analysis could be preferred when the data availability does not justify a richer statistical model application. On the contrary, for instance, when the analyst is estimating the extension of flood inundation for which both peak discharge and volume could play a similar role, a joint return period approach could be appealing. Indeed, an ensemble of equally rare scenarios (i.e. having the same return period) could be used to assess the variability of the obtained flood maps due to the selection of a single design event. Also in this case, it should be kept in mind that the univariate $n$ year return period is different to the bivariate and trivariate $n$ year return period. Even though it is to be expected that including more variables improves 
the modelling of the process, one should keep in mind the drastically increasing need of data to fit such models.

Acknowledgements. The critical and useful comments of two anonymous reviewers as well as G. Salvadori greatly improved this work, for which the authors are very grateful. The interested reader is referred to the public review process of this paper at HESSD for a discussion on JRP going beyond this publication. The research of the first author is partially funded by the German Research Foundation (DFG) under project PE 1632/4-1. S. Vandenberghe was a doctoral research fellow of the Research Foundation Flanders (FWO). The Special Research Fund of Ghent University financially enabled the research of M. J. van den Berg.

Edited by: N. Romano

\section{References}

Aas, K. and Berg, D.: Models for construction of multivariate dependence - a comparison study, Eur. J. Finance, 15, 639-659, doi:10.1080/13518470802588767, 2009.

Aas, K., Czado, C., Frigessi, A., and Bakken, H.: Pair-copula constructions of multiple dependence, Insur. Math. Econ., 44, 182198, doi:10.1016/j.insmatheco.2007.02.001, 2009.

Bedford, T. and Cooke, R.: Probability density decomposition for conditionally dependent random variables modeled by vines, Ann. Math. Artif. Intell., 32, 245-268, 2001.

Bedford, T. and Cooke, R.: Vines - A new graphical model for dependent random variables, Ann. Stat., 30, 1031-1068, 2002.

Berg, D.: Copula Goodness-of-fit testing: An overview and power comparison, Eur. J. Finance, 15, 675-701, 2009.

Brechmann, E. C. and Schepsmeier, U.: Modeling Dependence with C- and D-Vine Copulas: The R Package CDVine, J. Stat. Softw., 52, 1-27, 2013.

De Michele, C., Salvadori, G., Passoni, G., and Vezzoli, R.: A multivariate model of sea storms using copulas, Coast. Eng., 54, 734$751,2007$.

EEA: CORINE (Coordination of Information on Environment) Database, a key database for European integrated environmental assessment, Programme of the European Commission, 2000.

Favre, A. C., Adlouni, S. E., Perreault, L., Thiemonge, N., and Bobee, B.: Multivariate hydrological frequency analysis using copulas, Water Resour. Res., 40, W01101, doi:10.1029/2003WR002456, 2004.

Genest, C. and Favre, A. C.: Everything you always wanted to know about copula modeling but were afraid to ask, J. Hydrol. Eng., 12, 347-368, 2007.

Genest, C., Favre, A. C., Beliveau, J., and Jacques, C.: Metaelliptical copulas and their use in frequency analysis of multivariate hydrological data, Water Resour. Res., 43, W09401, doi:10.1029/2006WR005275, 2007.

Grimaldi, S. and Serinaldi, F.: Asymmetric copulas in multivariate flood frequency analysis, Adv. Water Resour., 29, 1155-1167, 2006a.

Grimaldi, S. and Serinaldi, F.: Design hydrograph analysis with 3copula function, Hydrol. Sci. J. - J. Sci. Hydrol., 51, 223-238, 2006b.
Grimaldi, S., Petroselli, A., Alonso, G., and Nardi, F.: Flow time estimation with variable hillslope velocity in ungauged basins, Adv. Water Resour., 33, 1216-1223, 2010.

Grimaldi, S., Petroselli, A., and Nardi, F.: A parsimonious geomorphological unit hydrograph for rainfall runoff modeling in small ungauged basins, Hydrol. Process., 57, 73-83, 2012a.

Grimaldi, S., Petroselli, A., and Romano, N.: Green-Ampt Curve Number mixed procedure as an empirical tool for rainfallrunoff modelling in small and ungauged basins, Hydrol. Process., doi:10.1002/hyp.9303, in press, 2012b.

Grimaldi, S., Petroselli, A., and Serinaldi, F.: Design hydrograph estimation in small and ungauged watersheds: continuous simulation method versus event-based approach, Hydrol. Process., 26, 3124-3134, 2012c.

Grimaldi, S., Petroselli, A., and Serinaldi, F.: A continuous simulation model for design-hydrograph estimation in ungauged watersheds, Hydrolog. Sci. J., 57, 1035-1051, 2012d.

Hobæk Haff, I., Aas, K., and Frigessi, A.: On the simplified paircopula construction - Simply useful or too simplistic?, J. Multivar. Anal., 101, 1296-1310, 2010.

IGMI: Raster (Matrix) numerical DEM of Italy, http://www.igmi. org/pdf/info_matrix2003.png, 26. Feb. 2013, 2003.

Joe, H.: Multivariate Models and Dependence Concepts, Chapman and Hall, 1997.

Kao, S.-C. and Govindaraju, R. S.: A bivariate frequency analysis of extreme rainfall with implications for design, J. Geophys. Res., 112, D13119, doi:10.1029/2007JD008522, 2007.

Kao, S.-C. and Govindaraju, R. S.: Trivariate statistical analysis of extreme rainfall events via the Plackett family of copulas, Water Resour. Res., 44, W02415, doi:10.1029/2007WR006261, 2008.

Kao, S.-C. and Govindaraju, R. S.: A copula-based joint deficit index for droughts, J. Hydrol., 380, 121-134, doi:10.1016/j.jhydrol.2009.10.029, 2010.

Kojadinovic, I. and Yan, J.: Modeling multivariate distributions with continuous margins using the copula $\mathrm{R}$ package, http://www. jstatsoft.org/v34/i09/, J. Stat. Softw., 34, 1-20, 2010.

Kurowicka, D. and Cooke, R. M.: Sampling algorithms for generating joint uniform distributions using the vine copula method, Comput. Stat. Data Anal., 51, 2889-2906, doi:10.1016/j.csda.2006.11.043, 2007.

Nelsen, R. B.: An Introduction to Copulas, Springer, 2006.

Panchenko, V.: Goodness-of-fit test for copulas, Physica A, 355, 176-182, 2005.

Pinya, M. A. S., Madsen, H., and Rosbjerg, D.: Assessment of the risk of inland flooding in a tidal sluice regulated catchment using multi-variate statistical techniques, Phys. Chem. Earth, 34, 662669, doi:10.1016/j.pce.2009.03.001, 2009.

R Core Team: R: A Language and Environment for Statistical Computing, R Foundation for Statistical Computing, http://www. R-project.org/, Vienna, Austria, 2011.

Salvadori, G.: Bivariate return periods via 2-copulas, J. Roy. Stat. Soc. Ser. B, 1, 129-144, 2004.

Salvadori, G. and De Michele, C.: Frequency analysis via copulas: theoretical aspects and applications to hydrological events, Water Resour. Res., 40, W12511, doi:10.1029/2004WR003133, 2004.

Salvadori, G. and De Michele, C.: Statistical characterization of temporal structure of storms, Adv. Water Resour., 29, 827-842, 2006. 
Salvadori, G. and De Michele, C.: On the use of copulas in hydrology: theory and practice, J. Hydrol. Eng., 12, 369-380, 2007.

Salvadori, G. and De Michele, C.: Multivariate Extreme Value Methods, in: Extremes in a Changing Climate, edited by: AghaKouchak, A., Easterling, D., Hsu, K., Schubert, S., and Sorooshian, S., Springer, 2013.

Salvadori, G., De Michele, C., Kottegoda, N., and Rosso, R.: Extremes in Nature, An Approach using Copulas, Springer, 2007.

Salvadori, G., De Michele, C., and Durante, F.: On the return period and design in a multivariate framework, Hydrol. Earth Syst. Sci., 15, 3293-3305, doi:10.5194/hess-15-3293-2011, 2011.

Schertzer, D. and Lovejoy, S.: Physical modeling and analysis of rain and clouds by anisotropic scaling multiplicative processes, J. Geophys. Res., 92, 9693-9714, 1987.

Serinaldi, F.: A multisite daily rainfall generator driven by bivariate copula-based mixed distributions, J. Geophys. Res.-Atmos., 114, D10103, doi:10.1029/2008JD011258, 2009.

Serinaldi, F.: Multifractality, imperfect scaling and hydrological properties of rainfall time series simulated by continuous universal multifractal and discrete random cascade models, Nonlin. Processes Geophys., 17, 697-714, doi:10.5194/npg-17-6972010, 2010.

Serinaldi, F.: Interactive comment on "Joint return periods in hydrology: a critical and practical review focusing on synthetic design hydrograph estimation" by S. Vandenberghe et al., Hydrol. Earth Syst. Sci. Discuss., 9, C3721-C3721, http://www.hydrol-earth-syst-sci-discuss.net/9/C3721/2012/ hessd-9-C3721-2012.png, 12 August 2012.

Serinaldi, F.: Closure to "Synthetic design hydrographs based on distribution functions with finite support" by F. Serinaldi and S. Grimaldi, J. Hydrol. Eng., 18, 126-129, 2013.

Serinaldi, F. and Grimaldi, S.: Fully nested 3-copula: procedure and application on hydrological data, J. Hydrol. Eng., 12, 420-430, 2007.
Serinaldi, F. and Grimaldi, S.: Synthetic design hydrographs based on distribution functions with finite support, J. Hydrol. Eng., 16, 434-446, doi:10.1061/(ASCE)HE.1943-5584.0000339, 2011.

Shiau, J. T.: Return period of bivariate distributed extreme hydrological events, Stoch. Environ. Res. Risk Assess., 17, 42-57, 2003.

Sklar, A.: Fonctions de répartition à $n$ dimensions et leurs marges, Publ. Inst. Statist. Univ. Paris, 8, 229-231, 1959.

Song, S. and Singh, V. P.: Meta-elliptical copulas for drought frequency analysis of periodic hydrologic data, Stoch. Environ. Res. Risk Assess., 24, 425-444, doi:10.1007/s00477-009-0331$1,2010$.

Theiling, C. H., and Burant, J. T.: Flood inundation mapping for integrated floodplain management: Upper Mississippi River system, River Res. Appl., doi:10.1002/rra.2583, in press, 2012.

Vandenberghe, S., Verhoest, N. E. C., Buyse, E., and De Baets, B.: A stochastic design rainfall generator based on copulas and mass curves, Hydrol. Earth Syst. Sci., 14, 2429-2442, doi:10.5194/hess-14-2429-2010, 2010.

Vandenberghe, S., Verhoest, N. E. C., Onof, C., and De Baets, B.: A comparative copula-based bivariate frequency analysis of observed and simulated storm events: A case study on BartlettLewis modeled rainfall, Water Resour. Res., 47, W07529, doi:10.1029/2009WR008388, 2011.

Volpi, E. and Fiori, A.: Design event selection in bivariate hydrological frequency analysis, Hydrolog. Sci. J., 57, 1 -10, 2012.

Wong, G., Lambert, M. F., Leonard, M., and Metcalfe, A. V.: Drought analysis using trivariate copulas conditional on climatic states, J. Hydrol. Eng., 15, 129-141, doi:10.1061/(ASCE)HE.1943-5584.0000169, 2010.

Yue, S. and Rasmussen, P.: Bivariate frequency analysis: discussion of some useful concepts in hydrological application, Hydrol. Process., 16, 2881-2898, 2002.

Zhang, L. and Singh, V. P.: Gumbel-Hougaard copula for trivariate rainfall frequency analysis, J. Hydrol. Eng., 12, 409-419, 2007. 